\title{
Efficacy and safety of intraventricular polymyxin B plus continuous ventricular drainage for the treatment of intracranial infection caused by drug-resistant Acinetobacter baumannii
}

\author{
Li Chen ${ }^{1}$, Xiyuan $\mathrm{Li}^{1}$, Dengkai $\mathrm{Li}^{1}$, Xuanwei Dong ${ }^{2}$, Hongwei Chen ${ }^{2}$ \\ ${ }^{1}$ Department of Intensive Care Unit, Aviation General Hospital, Beijing, China; ${ }^{2}$ Department of Neurosurgery for Cerebrospinal Fluid Diseases, \\ Aviation General Hospital, Beijing, China \\ Contributions: (I) Conception and design: L Chen, H Chen; (II) Administrative support: H Chen, X Li; (III) Provision of study materials or patients: \\ X Dong; (IV) Collection and assembly of data: L Chen, H Chen; (V) Data analysis and interpretation: L Chen, X Li, D Li, X Dong, H Chen; (VI) \\ Manuscript writing: All authors; (VII) Final approval of manuscript: All authors. \\ Correspondence to: Hongwei Chen. Department of Neurosurgery for Cerebrospinal Fluid Diseases, Aviation General Hospital, No. 3 Anwai Beiyuan, \\ Chaoyang District, Beijing 100012, China. Email: hongwei12@163.com.
}

\begin{abstract}
Background: The bacterial resistance rate has risen in recent years, and polymyxin $\mathrm{B}$ has been used more frequently to treat severe intracranial infection. This study aimed to investigate the clinical efficacy and safety profiles of intraventricular polymyxin B plus continuous ventricular drainage for the treatment of intracranial infection caused by drug-resistant Acinetobacter baumannii (DR-AB).

Methods: A retrospective study was performed on 21 patients who had an intracranial infection caused by DR-AB after neurosurgery at our hospital from May 2017 to July 2020. These patients were treated by intraventricular polymyxin B plus continuous ventricular drainage. The clinical features, treatment, cerebrospinal fluid (CSF)-related indicators, outcomes, and prognosis of these patients were analyzed.

Results: The external drainage tubes inserted into the lateral ventricle were kept unobstructed in all 21 patients. These patients received intraventricular polymyxin B $5 \mathrm{mg} /$ day plus intravenous antibiotics. The treatment with intraventricular polymyxin B lasted for $18.19 \pm 12.36$ days. The time to positive CSF culture was $10.50 \pm 10.60$ days. The bacterial clearance rate of CSF was $95.2 \%(20 / 21)$. The clinical cure rate was $81.0 \%(17 / 21)$, and the mortality rate was $19.0 \%$ (4/21). As for the causes of death, 1 case died from purulent CSF with cerebral abscess, which was considered to be caused by extensive brain parenchymal infection, 2 cases died from spontaneous intraventricular hemorrhage after returning negative for CSF cultures, and 1 case died from secondary massive cerebral infarction after returning negative for CSF cultures. There were no significant changes in the serum creatinine level before and after treatment.

Conclusions: For intracranial infection caused by DR-AB, early intraventricular polymyxin B plus continuous ventricular drainage could effectively clear the drug-resistant bacteria from CSF, thereby improving efficacy and reducing mortality. Renal functions before and after treatment were not changed significantly, proving that this combined treatment was safe and effective.
\end{abstract}

Keywords: Polymyxin B; continuous ventricular drainage; dug-resistant Acinetobacter baumannii (DR-AB); intracranial infection

Submitted May 14, 2021. Accepted for publication Jan 17, 2022.

doi: 10.21037/apm-21-3149

View this article at: https://dx.doi.org/10.21037/apm-21-3149 


\section{Introduction}

Acinetobacter baumannii is a gram-negative bacillus and is one of the main pathogens causing intracranial infections after neurosurgery. Drug-resistant Acinetobacter baumannii (DR-AB) are resistant to most or the vast majority of common antibiotics, and it is increasing year by year. $(1,2)$. Treating intracranial infection caused by Acinetobacter baumannii that is susceptible to only a few antibiotics poses a significant challenge, and is associated with increasingly higher mortality. Polymyxin B, a polypeptide antibiotic (3), was used to treat Gram-negative bacilli in the 1950s, and was later replaced by other antibiotics due to nephrotoxicity. Recently, polymyxin B has been used more frequently to cope with a yearly increase in the rate of bacterial resistance to antibiotics. It has been shown that only a tiny amount of intravenous polymyxin $\mathrm{B}$ can enter the cerebrospinal fluid (CSF) and exert an anti-infective effect $(4,5)$. Therefore, intraventricular and/or intrathecal administration may be more effective pathways to treat DR$\mathrm{AB}$-induced intracranial infection. Different from previous studies, this article only explores the efficacy and safety of intraventricular application of polymyxin B combined with continuous ventricular drainage technology in patients with DR-AB intracranial infection through retrospective analysis, and a detailed analysis of the cause of death for the future Provide basis for clinical work. We present the following article in accordance with the STROBE reporting checklist (available at https://apm.amegroups.com/article/ view/10.21037/apm-21-3149/rc).

\section{Methods}

\section{General data}

A total of 21 patients with intracranial infection caused by $\mathrm{DR}-\mathrm{AB}$ who received treatment at the Aviation General Hospital from May 2017 to July 2020 were recruited. The protocol was approved by the Medical Science Research Ethics Committee of the Aviation General Hospital (No. 2021-KY-01-10). The present study was retrospective and clinical data were collected from patients without interference of the treatment. Therefore, no physiological risks were brought to the patients. The data were collected and treated anonymously. After full discussion by the ethics committee, the patients were exempted from signing informed consent after weighing the risk-benefit ratio, and their confidentiality was protected. The present study followed the principles of the Declaration of Helsinki (as revised in 2013).

\section{Inclusion and exclusion criteria}

The inclusion criteria were as follows: (I) aged above 18 years old; (II) CSF cultures indicated DR-AB (6); (III) conforming to the diagnostic criteria for intracranial infection developed by the Chinese Neurosurgical Society of the Chinese Medical Association (7).

The exclusion criteria were as follows: (I) suspected of DR$\mathrm{AB}$ colonization or contamination; (II) history of intracranial infection before craniotomy; (III) previous treatment with polymyxin B due to intracranial infection or infection of other sites within 3 months; (IV) pregnancy or combined with malignancies outside the central nervous system.

\section{Treatment}

The treatment scheme was formulated individually after admission depending on whether the patients had the lumbar cistern drainage tube, ventriculoperitoneal shunt, or external drainage tube inserted into the lateral ventricle. CSF samples were collected for bacterial cultures. Intraventricular polymyxin B $5 \mathrm{mg} /$ day was administered immediately after the presence of DR-AB was indicated by the antimicrobial susceptibility test. (Because the lateral ventricle drainage technique was adopted for cerebrospinal fluid drainage in the cases of this study, polymyxin B was administered intracerebroventricularly).

The types and administration pathways of systemic antibiotics were as follows (depending on the results of the antimicrobial susceptibility test): polymyxin B $50 \mathrm{mg}$, IV drip, bid; tigecycline (ZeTan, Jiangsu Haosen Pharmaceutical Co, Jiangsu, China) $50 \mathrm{mg}$, IV drip, bid, after an initial dose of $100 \mathrm{mg}$; cefoperazone sodium and sulbactam sodium (Xianshu, Suzhou Dongrui Pharmaceutical Co., Ltd, Suzhou, China) $3 \mathrm{~g}$, IV drip, tid; piperacillin sodium and tazobactam sodium (North China pharmaceutical Group Corporation, Shijiazhuang, China) 2 g, IV drip, bid; amikacin (Dali Pharmaceutical Co., Ltd, Dali, China) 0.6 g, IV drip, bid; gentamicin (Shandong Xinhua Pharmaceutical Company Limited, Shandong, China) $80 \mathrm{mg}$, tid; etimicin sulfate (Xineng, Wuxi Jimin Shanhe Pharmaceutical Co., Ltd, Wuxi, China) $150 \mathrm{mg}$, bid.

\section{Determination of CSF-related indicators}

CSF analysis was conducted using the Hitachi LABOSPECT 008 AS Automatic Analyzer, and glucose, proteins, and chlorine were analyzed. The routine CSF 
parameters were analyzed manually since the instruments tended to overestimate the parameters. The BIOMERIEUX Vitek 2 Compact Bacteriological Analyzer was used for bacterial strain identification and the antimicrobial susceptibility test. The results of the antimicrobial susceptibility test were interpreted according to the Clinical and Laboratory Standards Institute (CLSI) antimicrobial susceptibility testing standards M100-S25 [2019]. The susceptibility to polymyxin B was evaluated based on the Food and Drug Administration (FDA) standards.

\section{Data collection}

Data were collected retrospectively, including age, gender, underlying diseases, principal diagnosis, name and time of craniocerebral operations before infection, type of systemic antibiotics, bacterial clearance of CSF, and clinical outcomes. Other laboratory test data collected before and after the treatment with polymyxin B included clinical manifestations, white blood cell count, red blood cell count, glucose, chlorine, protein, and liver and kidney function indicators.

\section{Efficacy evaluation}

Efficacy was divided into etiological and clinical efficacy. Etiological efficacy was considered if a patient was negative for CSF cultures 3 times consecutively after the treatment. Clinical efficacy was considered if a patient satisfied the following criteria: (I) normal body temperature for at least 3 consecutive days; (II) improvement or disappearance of the initial symptoms (meningeal irritation sign, consciousness); (III) CSF glucose above $2.2 \mathrm{mmol} / \mathrm{L} 3$ times consecutively, with the ratio of white blood cells to red blood cells $<1: 500$. Either was considered as clinical cure.

\section{Safety analysis}

Adverse events (AEs) related to medication were closely observed, including anemia, leukopenia, thrombocytopenia, hepatic and renal toxicity, diarrhea, and convulsion. The incidence of AEs was calculated.

\section{Statistical analysis}

Descriptive statistical analyses were performed using SPSS v.23.0 software. Continuous measurements were expressed as mean \pm standard deviation and medians [interquartile range $(\mathrm{IQR})]$. The data before and after treatment were compared using the paired t-test or Wilcoxon test. Enumeration data were expressed as IQR. $\mathrm{P}<0.05$ indicated a significant difference.

\section{Results}

\section{Baseline information of the patients}

A total of 21 patients were recruited, including 15 males and 6 females, with an average age of $46.33 \pm 16.96$ years old. All the included patients were referred to our hospital after being diagnosed with intracranial infection at other hospitals. They all had a history of neurosurgery before the infection. The primary diseases and the surgical procedures of these patients are listed in Table 1 .

\section{Surgical treatment and intracerebroventricular administration}

\section{Principles of surgical treatment}

The unblocked external drainage tubes inserted into the lateral ventricle upon admission were preserved; the lumbar cistern drainage tubes present upon admission were removed, and external drainage was performed for the lateral ventricle; for those without external drainage tubes inserted into the lateral ventricle upon admission, external drainage was performed for the lateral ventricle; all ventriculoperitoneal shunts upon admission were removed.

\section{Intraventricular administration}

The dose of intraventricular polymyxin B was $5 \mathrm{mg} /$ day in all patients. The treatment with intraventricular polymyxin B lasted for $18.19 \pm 12.36$ days. The time to positive CSF culture was $10.50 \pm 10.60$ days (Table 2).

\section{Results of bacterial testing and systemic medication}

The CSF cultures were positive for DR-AB in all patients. There were 6 cases infected with multidrug-resistant Acinetobacter baumannii (MDR-AB) and 15 cases with extensively drug-resistant Acinetobacter baumannii (XDR$\mathrm{AB})$. All patients were prescribed systemic antibiotics. The antibiotic schemes varied for different patients, and included tigecycline-cefoperazone sodium and sulbactam sodium $(n=8)$, tigecycline $(n=5)$, tigecycline-etimicin $(n=3)$, tigecycline-amikacin $(n=2)$, polymyxin-cefoperazone sodium and sulbactam sodium $(\mathrm{n}=1)$, tigecycline-polymyxin $(\mathrm{n}=1)$, tigecycline-piperacillin sodium and tazobactam 
Table 1 General information of the enrolled patients

\begin{tabular}{|c|c|}
\hline Patients & Outcome \\
\hline Total & 21 \\
\hline Average age (mean $\pm \mathrm{SD}$ ) (years) & $46.33 \pm 16.96$ \\
\hline \multicolumn{2}{|l|}{ Gender, n (\%) } \\
\hline Male & $15(71.4)$ \\
\hline Female & $6(28.6)$ \\
\hline \multicolumn{2}{|l|}{ Primary diagnosis, $\mathrm{n}(\%)$} \\
\hline Cerebral hemorrhage & $7(33.3)$ \\
\hline Traumatic brain injury & $6(28.6)$ \\
\hline Subarachnoid hemorrhage & $4(19.0)$ \\
\hline Brain tumor & $3(14.3)$ \\
\hline Lupus encephalitis & $1(4.8)$ \\
\hline Operation history before infection, $\mathrm{n}(\%)$ & $21(100.0)$ \\
\hline \multicolumn{2}{|l|}{ Surgical procedures before infection, n (\%) } \\
\hline LD & $16(76.2)$ \\
\hline EVD & $13(61.9)$ \\
\hline$\geq 2$ times LD, $\mathrm{n}(\%)$ & $6(28.6)$ \\
\hline$\geq 2$ times EVD, n (\%) & $5(23.8)$ \\
\hline Craniotomy, n (\%) & $14(66.7)$ \\
\hline Evacuation of intracranial hematoma, n (\%) & $5(23.8)$ \\
\hline Decompressive craniectomy, n (\%) & 7 (33.3) \\
\hline VPS, n (\%) & $1(4.8)$ \\
\hline Intracranial space occupying resection, n (\%) & $3(14.3)$ \\
\hline Ventriculoscopic surgery, n (\%) & $1(4.8)$ \\
\hline Interventional surgery for aneurysm, n (\%) & $3(14.3)$ \\
\hline Aneurysm clipping surgery, $\mathrm{n}(\%)$ & $1(4.8)$ \\
\hline
\end{tabular}

LD, lumbar-cistern drainage; EVD, external ventricular drainage;

VPS, ventriculoperitoneal shunt. sodium $(\mathrm{n}=1)$, tigecycline-gentamicin $(\mathrm{n}=1)$, and amikacin $(\mathrm{n}=1)$. The average duration of systemic antibiotic use was $42.0 \pm 27.4$ days.

\section{Clinical symptoms before and after treatment and CSF test data}

Clinical symptoms before and after treatment and CSF test data are shown in Table 3.

\section{Treatment results and complications}

The bacterial clearance rate of CSF was 95.2\% (20/21) in the 21 patients. The clinical cure rate was $81.0 \%$ $(17 / 21)$, and the mortality rate was $19.0 \%(4 / 21)$. The causes of death were as follows: 2 cases had spontaneous intraventricular hemorrhage after returning negative for CSF cultures, and the disease deteriorated. Both died after they gave up treatment and returned home. One case already had purulent CSF with multiple brain abscesses upon admission. This case was suspected to have extensive brain parenchymal infection. Although the CSF cultures returned as negative after using intraventricular polymyxin $\mathrm{B}$, the fluid drained from the abscess cavity was repeatedly positive. This patient gave up treatment and died after returning home. One case had secondary massive cerebral infarction after the CSF culture returned as negative. This patient gave up treatment and died after returning home. The patients who were cured were followed up for 4 months to 3 years. None of the patients relapsed (Table 4).

\section{Safety evaluation}

Before and after the treatment with intraventricular

Table 2 Surgical treatment of intracranial infection and intraventricular/intrathecal administration

\begin{tabular}{lc}
\hline Surgical treatment and intraventricular/intrathecal administration & Outcome \\
\hline EVD, $\mathrm{n}(\%)$ & $18(85.7)$ \\
Reserve original EVD, $\mathrm{n}(\%)$ & $7(33.3)$ \\
Remove LD, $\mathrm{n}(\%)$ & $1(4.8)$ \\
Remove VPS, $\mathrm{n}(\%)$ & $1(4.8)$ \\
Polymyxin B-intraventricular administration, $\mathrm{n}(\%)$ & $21(100.0)$ \\
Dosage for polymyxin B, 5 mg/day, $\mathrm{n}(\%)$ & $21(100.0)$ \\
Course of treatment for polymyxin B intraventricular administration, (mean \pm SD) (days) & $18.19 \pm 12.36$ \\
\hline
\end{tabular}

EVD, external ventricular drainage; LD, lumbar-cistern drainage; VPS, ventriculoperitoneal shunt. 
Table 3 Clinical symptoms and laboratory data of the 21 patients

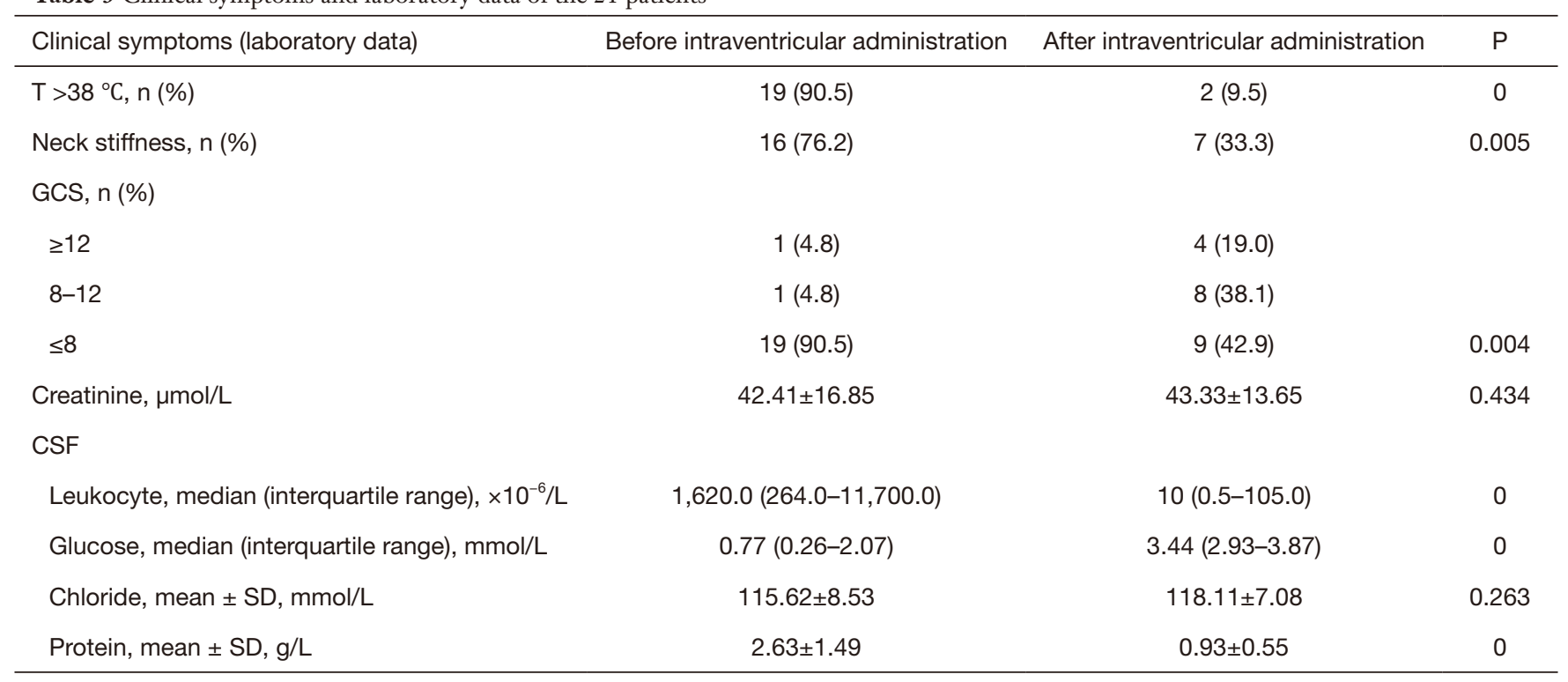

GCS, Glasgow Coma Scale; CSF, cerebrospinal fluid.

Table 4 Outcome of the 21 patients

\begin{tabular}{lc}
\hline Outcome & Data \\
\hline Clinical cure rate, $\mathrm{n}(\%)$ & $17(81.0)$ \\
Pathogen clearance rate, $\mathrm{n}(\%)$ & $20(95.2)$ \\
CSF bacteria negative time, mean $\pm \mathrm{SD}$ (days) & $10.50 \pm 10.60$ \\
Death, $\mathrm{n}(\%)$ & $4(19.0)$ \\
Cerebral hemorrhage & $2(9.5)$ \\
Brain parenchymal infection & $1(4.8)$ \\
Large cerebral infarction & $1(4.8)$ \\
\hline
\end{tabular}

CSF, cerebrospinal fluid.

polymyxin $B$, the serum creatinine levels were $42.41 \pm 16.85$ and $43.33 \pm 13.65 \mu \mathrm{mol} / \mathrm{L}$, respectively, indicating no significant difference $(\mathrm{P}=0.434)$. No kidney function impairment was observed.

\section{Discussion}

Intracranial infection is a common and severe complication after neurosurgery $(8,9)$, which may lead to prolonged hospital stay, increased medical costs, or even death. The incidence of intracranial infection varies between $0.72 \%$ and $8 \%(10)$. Intracranial infection most often occurs after craniocerebral operations. External ventricular drainage and lumbar cistern drainage after surgeries for intracranial tumors, severe craniocerebral injury, cerebroventricular hemorrhage, subarachnoid hemorrhage, and hydrocephalus may lead to the damage of the blood-brain barrier and the decline of immune system function in critically ill patients, which are the leading causes of intracranial infection (11). All patients included in the present study had a history of neurosurgery, typically lumbar cistern drainage, external ventricular drainage, and craniotomy. The main pathogens of intracranial infection were identified as Gram-positive cocci and Gram-negative bacilli. The AB-induced infection rate after neurosurgery is as high as $15-21.74 \%$ (2). The incidence of intracranial infection and the prevalence of drug-resistant bacteria continue to rise, which poses a challenge to clinical treatment.

Common medications against $\mathrm{Ab}$-induced infections include sulbactam, cephalosporin, carbapenems, $\beta$-lactams, aminoglycosides, fluoroquinolones, tetracyclines, and polymyxin. However, only a limited number of antibiotics are potent against DR-AB. Upon admission, the antimicrobial resistance rates for carbapenems, cephalosporins, and aminoglycosides reached up to $95.2 \%$, $100 \%$, and $90.5 \%$ among our patients, respectively, which might be related to the prior use of large-dose high-grade antibiotics at other hospitals. DR-AB strains are known for their extremely high drug resistance, which is related to treatment difficulty and poor prognosis. It is urgent to look 
for antibiotics that are unlikely to cause drug resistance.

Polymyxin B is a rapid-acting bactericidal agent, which exhibits good activity against the vast majority of Gram-negative bacteria (12). It was once replaced by safer antibiotics due to its narrow antibacterial spectrum and significant nephrotoxicity. In recent years, infections caused by multidrug-resistant Gram-negative bacteria have become widespread worldwide, which has necessitated the reemergence of polymyxin B (13). It has been shown that polymyxin $B$ is effective against various severe nosocomial infections caused by $\mathrm{Ab}$, including bacteremia, urinary tract infection, and pulmonary infections (14). However, polymyxin $\mathrm{B}$ can hardly penetrate the blood-brain barrier to reach an effective inhibitory concentration in CSF due to its high molecular weight. Few reports have been published at home and abroad regarding the use of polymyxin $\mathrm{B}$ in intracranial infection.

According to the Chinese Expert Consensus on the Diagnosis and Treatment of Infection in Neurosurgical Critically Ill Patients [2017] developed by the Chinese Neurosurgical Society of the Chinese Medical Association and the Chinese Collaboration Group for Infection Diagnosis and Treatment in Neurosurgical Critically Ill Patients in 2017 (7), intraventricular/intrathecal administration is effective for treating intracranial infection, especially when patients are unresponsive to systemic intravenous antibiotics. The above administration pathway bypasses the blood-brain barrier and directly delivers the antibiotics to the sites of infection. Therefore, the drugs can work more directly and effectively while preventing the toxic reactions associated with systemic antibiotic use (15). Given the facts above, intraventricular/intrathecal polymyxin $\mathrm{B}$ may be a good alternative for the treatment of DR$\mathrm{AB}$-induced intracranial infection. It has been reported that the use of polymyxin B alone may be related to poor outcomes (16). In contrast, the combined use of other antibiotics can significantly enhance the effectiveness and reduce mortality (17). At present, there has been no uniform standard for the treatment cycle in patients with severe intracranial infection. The treatment may last for as long as 4-8 weeks. We recruited a total of 21 patients with $\mathrm{DR}-\mathrm{AB}$-induced intracranial infection after neurosurgeries, all of which received intraventricular polymyxin B for $18.19 \pm 12.36$ days and combined intravenous administration of several sensitive antibiotics for $51.14 \pm 29.68$ days. The bacterial clearance rate of CSF reached up to $95.2 \%(20 / 21)$, and the clinical cure rate was $81.0 \%(17 / 21)$. The results showed that intraventricular polymyxin B could effectively eliminate DR-AB from CSF, working synergistically with intravenous antibiotics.

According to a recent report, the mortality of DR-ABinduced intracranial infection was markedly high, reaching up to $40.3 \%(18)$. In our study, the overall mortality was $19.0 \%(4 / 21)$. The mortality directly caused by the infection was $1 / 21$ (4.8\%). Both were lower than the levels reported in the literature. This might be due to the early use of intraventricular polymyxin B after the discovery of DR-AB. Wang et al. also reported that early, effective intraventricular drug administration could improve the cure rate of intracranial infections after neurosurgeries (19).

However, polymyxin B has considerable nephrotoxicity, neurotoxicity, and neuromuscular blocking effects. One recent study demonstrated that the incidence and severity of nephrotoxicity associated with polymyxin B were actually low (20). Besides, these side effects were reversible after discontinuation (21). During treatment, these AEs did not affect any of our patients. The serum creatinine levels before and after treatment were $42.41 \pm 16.85$ and $43.33 \pm$ $13.65 \mu \mathrm{mol} / \mathrm{L}$, respectively, without a significant difference $(\mathrm{P}>0.05)$. Thus, the treatment scheme had a favorable safety profile for DR-AB-induced intracranial infection.

In addition to intracerebroventricular administration, the necessary surgical methods are particularly important in the treatment of intracranial infections. For example, early removal of infection-related artificial materials $(7,22)$ and continuous cerebrospinal fluid drainage $(23)$. Ventricular drainage and lumbar cistern drainage are currently commonly used methods of cerebrospinal fluid drainage. Some scholars believe that lumbar cistern drainage can better reduce the recurrence of intracranial infections. The lumbar cistern drainage tubes are generally narrow. The DR-AB-infected CSF is turbid, contains floccules, and may even become purulent, blocking the drainage tube and increasing the risk of infection. The previous research of our research team showed that the extraventricular drainage tube may be more suitable for drainage of purulent cerebrospinal fluid due to its thicker tubing (24). Among the 21 patients in the present study, 1 patient had a lumber cistern drainage tube and 1 patient had a ventriculoperitoneal shunt upon admission. Both were removed after admission. Another 7 patients did not have their external ventricular drainage tubes removed upon admission since the tubes were maintained unblocked and were later used to drain inflammatory CSF and to administer the drugs intraventricularly. These drainage tubes were removed after the infection was controlled 
properly. Overall, continuous, unblocked CSF drainage can effectively remove the bacteria-infected CSF outside the body. The drainage tubes should not be replaced or removed prematurely or frequently to avoid secondary injury to brain tissues and the central nervous system, thereby reducing intracranial hemorrhage.

\section{Conclusions}

For DR-AB-induced intracranial infection, early treatment with intraventricular polymyxin B plus continuous external ventricular drainage could effectively clear DR-AB from CSF, thereby improving efficacy and reducing mortality. There were no significant changes in renal function before and after treatment, indicating a favorable safety profile of this treatment scheme. However, these findings need to be further corroborated in large-scale studies in the future.

Our study also had the following limitations: we did not comprehensively analyze the antibacterial activity of polymyxin $\mathrm{B}$, drug concentration in CSF, or optimal drug dose for DR-AB-induced intracranial infection.

\section{Acknowledgments}

Funding: We acknowledge the support received from Scientific Research Project of Aviation General Hospital of Beijing (YJ202021).

\section{Footnote}

Reporting Checklist: The authors have completed the STROBE reporting checklist. Available at https://apm. amegroups.com/article/view/10.21037/apm-21-3149/rc

Data Sharing Statement: Available at https://apm.amegroups. com/article/view/10.21037/apm-21-3149/dss

Conflicts of Interest: All authors have completed the ICMJE uniform disclosure form (available at https://apm. amegroups.com/article/view/10.21037/apm-21-3149/coif). The authors have no conflicts of interest to declare.

Ethical Statement: The authors are accountable for all aspects of the work in ensuring that questions related to the accuracy or integrity of any part of the work are appropriately investigated and resolved. The present study followed the principles of the Declaration of Helsinki (as revised in 2013). This study has been approved by the
Medical Science Research Ethics Committee of Aviation General Hospital (No. 2021-KY-01-10). The patients were exempted from signing informed consent.

Open Access Statement: This is an Open Access article distributed in accordance with the Creative Commons Attribution-NonCommercial-NoDerivs 4.0 International License (CC BY-NC-ND 4.0), which permits the noncommercial replication and distribution of the article with the strict proviso that no changes or edits are made and the original work is properly cited (including links to both the formal publication through the relevant DOI and the license). See: https://creativecommons.org/licenses/by-nc-nd/4.0/.

\section{References}

1. Ruan L, Wu D, Li X, et al. Analysis of microbial community composition and diversity in postoperative intracranial infection using high-throughput sequencing. Mol Med Rep 2017;16:3938-46.

2. Jiang L, Guo L, Li R, et al. Targeted surveillance and infection-related risk factors of nosocomial infection in patients after neurosurgical operation. Pak J Pharm Sci 2017;30:1053-6.

3. Magiorakos AP, Srinivasan A, Carey RB, et al. Multidrugresistant, extensively drug-resistant and pan-drug-resistant bacteria: an international expert proposal for interim standard definitions for acquired resistance. Clin Microbiol Infect 2012;18:268-81.

4. Antachopoulos C, Karvanen M, Iosifidis E, et al. Serum and cerebrospinal fluid levels of colistin in pediatric patients. Antimicrob Agents Chemother 2010;54:3985-7.

5. Markantonis SL, Markou N, Fousteri M, et al. Penetration of colistin into cerebrospinal fluid. Antimicrob Agents Chemother 2009;53:4907-10.

6. $\mathrm{Li} \mathrm{CH}, \mathrm{Wu}$ Anhua. Interim standard definitions of MDR, XDR, and PDR multi-drug resistant bacte-riainternational expert advice. Chinese Journal of Infection Control 2014;13:62-4.

7. Neurosurgery Branch of Chinese Medical Association,China Neurosurgery Critical Care Management Cooperative Group. Expert consensus on diagnosis and treatment of infection in critically ill patients in Chinese neurosurgery (2017). National Medical Journal of China 2017;97:1607-14.

8. Moazzam AA, Rajagopal SM, Sedghizadeh PP, et al. Intracranial bacterial infections of oral origin. J Clin Neurosci 2015;22:800-6. 
9. Golan E, Wong K, Alahmadi H, et al. Endoscopic sphenoid sinus drainage in Lemierre syndrome. J Clin Neurosci 2014;21:346-8.

10. Zhan R, Zhu Y, Shen Y, et al. Post-operative central nervous system infections after cranial surgery in China: incidence, causative agents, and risk factors in 1,470 patients. Eur J Clin Microbiol Infect Dis 2014;33:861-6.

11. Cui XL, Lin S, Sui DL, et al. Progress in diagnosis and treatment of intracranial infection after neurosurgery. Chinese Journal of Neurosurgery 2014;30:312-4.

12. Neiva LB, Borges FT, Watanabe M, et al. Nephrotoxicity of polymyxin B: experimental study in cells and implications for nursing practice. Rev Esc Enferm USP 2014;48:272-7.

13. Li J, Nation RL, Turnidge JD, et al. Colistin: the reemerging antibiotic for multidrug-resistant Gram-negative bacterial infections. Lancet Infect Dis 2006;6:589-601.

14. Chinese Research Hospital Association Of Critical Care Medicine, Chinese Research Hospital Association Of Evidence Base And Translational Infectious Diseases. Chinese expert consensus on polymyxins in the clinical practice. Zhonghua Wei Zhong Bing Ji Jiu Yi Xue 2019;31:1194-8.

15. Wen DY, Bottini AG, Hall WA, et al. Infections in neurologic surgery. The intraventricular use of antibiotics. Neurosurg Clin N Am 1992;3:343-54.

16. Nadkarni AS, Schliep T, Khan L, et al. Cluster of bloodstream infections caused by KPC-2 car-bapenemaseproducing Klebsiella pneumoniae in Manhattan. Am J Infect Control 2009;37:121-6.

17. Cai B, Cai Y, Liew YX, et al. Clinical Efficacy of Polymyxin Monotherapy versus Nonvalidated Polymyxin Combination Therapy versus Validated Polymyxin Combination Therapy in Extensively Drug-Resistant

Cite this article as: Chen L, Li X, Li D, Dong X, Chen H. Efficacy and safety of intraventricular polymyxin B plus continuous ventricular drainage for the treatment of intracranial infection caused by drug-resistant Acinetobacter baumannii. Ann Palliat Med 2022;11(2):490-497. doi: 10.21037/apm-21-3149
Gram-Negative Bacillus Infections. Antimicrob Agents Chemother 2016;60:4013-22.

18. Sharma R, Goda R, Borkar SA, et al. Outcome following postneurosurgical Acinetobacter meningitis: an institutional experience of 72 cases. Neurosurg Focus 2019;47:E8.

19. Wang JH, Lin PC, Chou CH, et al. Intraventricular antimicrobial therapy in postneurosurgical Gramnegative bacillary meningitis or ventriculitis: a hospitalbased retrospective study. J Microbiol Immunol Infect 2014;47:204-10.

20. Shahbazi F, Dashti-Khavidaki S. Colistin: efficacy and safety in different populations. Expert Rev Clin Pharmacol 2015;8:423-48.

21. Nelson BC, Eiras DP, Gomez-Simmonds A, et al. Clinical outcomes associated with polymyxin $\mathrm{B}$ dose in patients with bloodstream infections due to carbapenem-resistant Gram-negative rods. Antimicrob Agents Chemother 2015;59:7000-6.

22. Tunkel AR, Hasbun R, Bhimraj A, et al. 2017 Infectious Diseases Society of America's Clinical Practice Guidelines for Healthcare-Associated Ventriculitis and Meningitis. Clin Infect Dis 2017;64:e34-65.

23. Shang F, Xu Y, Wang N, et al. Diagnosis and treatment of severe neurosurgical patients with pyogenic ventriculitis caused by gram-negative bacteria. Neurol Sci 2018;39:79-84.

24. Chen H, Guo X, Xie D, et al. A Clinical Study on the Use of Intraventricular Polymyxin B Supplemented by Continuous External Ventricular Drainage in the Treatment of Drug-Resistant Gram-Negative Bacilli Intracranial Infection. Infect Drug Resist. 2020;13:2963-70.

(English Language Editor: C. Betlazar-Maseh) 